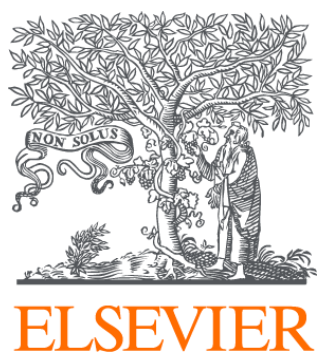

Since January 2020 Elsevier has created a COVID-19 resource centre with free information in English and Mandarin on the novel coronavirus COVID-

19. The COVID-19 resource centre is hosted on Elsevier Connect, the company's public news and information website.

Elsevier hereby grants permission to make all its COVID-19-related research that is available on the COVID-19 resource centre - including this research content - immediately available in PubMed Central and other publicly funded repositories, such as the WHO COVID database with rights for unrestricted research re-use and analyses in any form or by any means with acknowledgement of the original source. These permissions are granted for free by Elsevier for as long as the COVID-19 resource centre remains active. 


\section{Transmission of COVID-19 in 282 clusters in Catalonia, Spain: a cohort study}

Michael Marks, Pere Millat-Martinez, Dan Ouchi, Chrissy h Roberts, Andrea Alemany, Marc Corbacho-Monné, Maria Ubals, Aurelio Tobias, Cristian Tebé, Ester Ballana, Quique Bassat, Bàrbara Baro, Martí Vall-Mayans, Camila G-Beiras, Nuria Prat, Jordi Ara, Bonaventura Clotet, Oriol Mitjà

\section{Summary}

Background Scarce data are available on what variables affect the risk of transmission of severe acute respiratory syndrome coronavirus 2 (SARS-CoV-2), the development of symptomatic COVID-19, and, particularly, the relationship with viral load. We aimed to analyse data from linked index cases of COVID-19 and their contacts to explore factors associated with transmission of SARS-CoV-2.

Methods In this cohort study, patients were recruited as part of a randomised controlled trial done between March 17 and April 28, 2020, that aimed to assess if hydroxychloroquine reduced transmission of SARS-CoV-2. Patients with COVID-19 and their contacts were identified by use of the electronic registry of the Epidemiological Surveillance Emergency Service of Catalonia (Spain). Patients with COVID-19 included in our analysis were aged 18 years or older, not hospitalised, had quantitative PCR results available at baseline, had mild symptom onset within 5 days before enrolment, and had no reported symptoms of SARS-CoV-2 infections in their accommodation or workplace within the 14 days before enrolment. Contacts included were adults with a recent history of exposure and absence of COVID-19-like symptoms within the 7 days preceding enrolment. Viral load of contacts, measured by quantitative PCR from a nasopharyngeal swab, was assessed at enrolment, at day 14, and whenever the participant reported COVID-19-like symptoms. We assessed risk of transmission and developing symptomatic disease and incubation dynamics using regression analysis. We assessed the relationship of viral load and characteristics of cases (age, sex, number of days from reported symptom onset, and presence or absence of fever, cough, dyspnoea, rhinitis, and anosmia) and associations between risk of transmission and characteristics of the index case and contacts.

Findings We identified 314 patients with COVID-19, with 282 (90\%) having at least one contact (753 contacts in total) resulting in 282 clusters. 90 (32\%) of 282 clusters had at least one transmission event. The secondary attack rate was $17 \%$ (125 of 753 contacts), with a variation from $12 \%$ when the index case had a viral load lower than $1 \times 10^{6}$ copies per $\mathrm{mL}$ to $24 \%$ when the index case had a viral load of $1 \times 10^{10}$ copies per $\mathrm{mL}$ or higher (adjusted odds ratio per $\log _{10}$ increase in viral load 1·3, 95\% CI 1·1-1·5). Increased risk of transmission was also associated with household contact $(3 \cdot 0,1 \cdot 59-5 \cdot 65)$ and age of the contact (per year: 1.02,1·01-1.04). 449 contacts had a positive PCR result at baseline. $28(6 \%)$ of 449 contacts had symptoms at the first visit. Of 421 contacts who were asymptomatic at the first visit, 181 (43\%) developed symptomatic COVID-19, with a variation from approximately $38 \%$ in contacts with an initial viral load lower than $1 \times 10^{7}$ copies per $\mathrm{mL}$ to greater than $66 \%$ for those with an initial viral load of $1 \times 10^{10}$ copies per $\mathrm{mL}$ or higher (hazard ratio per $\log _{10}$ increase in viral load $1 \cdot 12,95 \%$ CI $1 \cdot 05-1 \cdot 20 ; p=0 \cdot 0006$ ). Time to onset of symptomatic disease decreased from a median of 7 days (IQR 5-10) for individuals with an initial viral load lower than $1 \times 10^{7}$ copies per $\mathrm{mL}$ to 6 days (4-8) for those with an initial viral load between $1 \times 10^{7}$ and $1 \times 10^{9}$ copies per $\mathrm{mL}$, and 5 days (3-8) for those with an initial viral load higher than $1 \times 10^{9}$ copies per $\mathrm{mL}$.

Interpretation In our study, the viral load of index cases was a leading driver of SARS-CoV-2 transmission. The risk of symptomatic COVID-19 was strongly associated with the viral load of contacts at baseline and shortened the incubation time of COVID-19 in a dose-dependent manner.

Funding YoMeCorono, Generalitat de Catalunya.

Copyright (C) 2021 Elsevier Ltd. All rights reserved.

\section{Introduction}

According to current evidence, COVID-19 is primarily transmitted from person to person through respiratory droplets, as well as indirect contact through transfer of the virus from contaminated fomites to the mouth, nose, or eyes. ${ }^{1,2}$ As with most respiratory viral infections, some transmission through smaller aerosols is likely to occur, but their relative contribution compared with droplets remains unclear. Several outbreak investigation reports have shown that COVID-19 transmission can be particularly effective in confined indoor spaces such as workplaces, including factories, churches, restaurants, shopping centres, and healthcare settings. ${ }^{3-6}$ In Spain and many other countries, health-care workers have had a high rate of COVID-19 infection?
Lancet Infect Dis 2021
Published Online
February 2, 2021
https://doi.org/10.1016/
S1473-3099(20)30985-3
See Online/Comment
https://doi.org/10.1016/
S1473-3099(21)00005-0

For the Catalan translation of the abstract see Online for appendix 1

Clinical Research Department, Faculty of Infectious \& Tropical Diseases, London School of Hygiene \& Tropical Medicine, London, UK (M Marks PhD, ( h Roberts PhD); Hospital for Tropical Diseases, University College London Hospital, London, UK (M Marks); Barcelona Institute for Global Health, Hospital Clínic, University of Barcelona, Barcelona, Spain (P Millat-Martinez MD, Prof Q Bassat PhD, B Baro PhD); IrsiCaixa AIDS Research Institute, Badalona, Spain (D Ouchi MSc, E Ballana PhD, $B$ (lotet PhD); Fight AIDS and Infectious Diseases Foundation, Badalona, Spain (A Alemany BM,

$M$ Corbacho-Monné BM, M Vall-Mayans PhD,

C G-Beiras PhD, B Clotet, O Mitjà PhD); Parc Taulí Hospital Universitari, Institut d'Investigació i Innovació Parc Taulí I3PT, Sabadell, Spain (M Corbacho-Monné); Catalan Institution for Research and Advanced Studies, Barcelona, Spain (Prof Q Bassat); Infectious Disease Department, Hospital Universitari Germans Trias i Pujol, Badalona, Spain (M Ubals MD, E Ballana, M Vall-Mayans, N Prat MSc, J Ara PhD, B Clotet, O Mitjà); Institute of Environmental Assessment and Water Research, Spanish Council for Scientific Research, Barcelona Spain (A Tobias PhD); Biostatistics Unit, Institut d'Investigació Biomèdica de Bellvitge, L'Hospitalet de Llobregat, Barcelona, Spain (CTebé PhD); Department of Clinical 
Sciences, Faculty of Medicine and Health Sciences, University of Barcelona, Barcelona, Spain (C Tebé, O Mitjà); Càtedra de Malalties Infeccioses i Immunitat, Universitat de Vic, Universtiat Central de Catalunya, Vic, Spain (B Clotet, $O$ Mitjà); Lihir Medical Centre, International SOS, Lihir Island,

Papua New Guinea (O Mitjà) Correspondence to: Dr Michael Marks, Clinical Research Department, London School of Hygiene \& Tropical Medicine, London WC1E 7HT, UK michael.marks@lshtm.ac.uk
Research in context

\section{Evidence before this study}

On Sept 20, 2020, we searched PubMed for articles reporting on factors influencing transmission of severe acute respiratory syndrome coronavirus 2 (SARS-CoV-2) and the risk of developing symptomatic COVID-19. Search terms included "COVID-19", "SARS-CoV-2", "transmission", "incubation time", and "risk", with no language restrictions. Various authors had reported on retrospective analyses of clusters of index cases and their corresponding contacts, as well as series of patients who developed symptomatic COVID-19 after a positive PCR result. Besides describing the secondary attack rate, these studies identified risk factors for transmission associated with the place and duration of exposure and not using personal protective equipment. A single study suggested that individuals who were symptomatic might be more likely to transmit than those without symptoms, but we found no clear evidence regarding the influence of viral load of the index case on transmission risk. Similarly, although various retrospective series of patients with positive PCR results had reported incubation times elsewhere, the characteristics of index case and contacts that might influence the risk of developing symptomatic COVID-19 and the time to this event had been barely addressed.

\section{Added value of this study}

We analysed data from a large cluster-randomised clinical trial on post-exposure therapy for COVID-19 that provided new information on SARS-CoV-2 transmission dynamics. Several design components add value to this dataset. Notably, quantitative $P C R$ was available for the index cases to estimate risk of transmission. Additionally, quantitative PCR was also done on asymptomatic contacts at the time of enrolment allowing us to investigate the dynamics of symptomatic disease onset among them. We found that the viral load of the index case was the leading determinant of the risk of SARS-CoV-2 PCR positivity among contacts. Among contacts who were SARS-CoV-2 $P C R$ positive at baseline, viral load significantly influenced the risk of developing the symptomatic disease in a dose-dependent manner. This influence also became apparent in the incubation time, which shortened with increasing baseline viral loads.

\section{Implications of all the available evidence}

Our results provide important insights into the knowledge regarding the risk of SARS-CoV-2 transmission and COVID-19 development. The fact that the transmission risk was primarily driven by the viral load of index cases, more than other factors such as their symptoms or age, suggests that all cases should be considered potential transmitters irrespective of their presentation and encourages the assessment of viral load in patients with a larger number of close contacts. Similarly, our results regarding the risk and expected time to developing symptomatic COVID-19 encourage risk stratification of newly diagnosed SARS-CoV-2 infections on the basis of the initial viral load.
The availability of data regarding the factors that might enhance transmission is essential for designing interventions to control the spread of severe acute respiratory syndrome coronavirus 2 (SARS-CoV-2). Available data provide information on the risk of transmission related to the place and duration of exposure and the use of respiratory and eye protection, ${ }^{1,3-5,8}$ but not on other factors related to the characteristics of index cases and their contacts. Over the course of infection, the virus has been identified in respiratory tract specimens 1-2 days before the onset of symptoms, and it can persist for prolonged periods, over several weeks after the onset of symptoms in mild cases. ${ }^{9}$ However, the detection of viral RNA by PCR does not necessarily equate with infectivity, and the exact relationship between viral load and risk of transmission from a case is still not clear. ${ }^{10,11}$ Studies investigating case-contact pairs have reported highly variable secondary attack rates (ie, ranging from $0.7 \%$ to $75 \%$ ), depending on the type of exposureduration, place, pre-spymptomatic or post-symptomatic. ${ }^{12-15}$

Another challenge for public health interventions is the risk stratification of individuals who are infected for developing symptomatic illness. A living systematic review estimated that the proportion of PCR-positive infected contacts that progress to symptomatic disease is approximately $70-80 \% .^{16,17}$ Mean or median incubation period has been consistently estimated to be between 5 to 7 days. ${ }^{18-20}$ Although studies have suggested that the viral load of cases might be associated with risk of disease or transmission, no published data so far have directly addressed this question, and little is known about factors that might contribute to variation on the risk of developing COVID-19 symptoms or the incubation periods among individuals who are infected.

The overall aim of this study was to evaluate transmission dynamics of SARS-CoV-2 in the context of a trial of postexposure prophylaxis. Specifically, the objectives of our study were threefold: to investigate the association between clinical and demographic features of cases and viral load, to evaluate the effect of viral load on SARS-CoV-2 transmission to close contacts, and to determine the influence of viral load in the exposed individuals on development of symptoms and on the incubation period.

\section{Methods \\ Study design}

This study was a post-hoc analysis of data collected in the BCN PEP CoV-2 Study (NCT04304053), a clusterrandomised trial that included individuals with PCRconfirmed COVID-19 and their close contacts. The trial occurred between March 17 and April 28, 2020, during the initial wave of the SARS-CoV-2 outbreak, in three of 
nine health-care areas in Catalonia (northeast Spain)Catalunya Central, Àmbit Metropolità Nord, and Barcelona Ciutat - with a total target population of 4206440 people. The study protocol of the BCN PEP CoV-2 Study was approved by the ethics committee of Hospital Germans Trias Pujol (Badalona, Spain). Written informed consent was obtained from all participants. Full details of the original study are reported elsewhere. ${ }^{21}$

COVID-19 cases were identified by use of the electronic registry of the Epidemiological Surveillance Emergency Service of Catalonia of the Department of Health. ${ }^{22}$ Following government ordinance, the surveillance service registered all new COVID-19 diagnoses that occurred from March 16, 2020 onwards. The surveillance system included active tracing of all contacts with recent history of exposure, defined as being in contact with a case of SARS-CoV-2 confirmed by PCR within $2 \mathrm{~m}$ of distance for more than 15 min.

\section{Study participants}

All patients with COVID-19 included in our analysis were adults (aged 18 years or older) who were not hospitalised and had quantitative PCR results available at baseline, mild symptom onset within 5 days before enrolment, and no reported symptoms of SARS-CoV-2 infections in their accommodation (ie, household or nursing home) or workplace within the 14 days before enrolment. Contacts selected for the analysis were adults with a recent history of exposure and absence of COVID-19-like symptoms within the 7 days preceding enrolment. Contacts were exposed to the index case as either a health-care worker, a household contact, a nursing home worker, or a nursing home resident.

We selected all eligible individuals within the original trial population for each of the three analyses done in this study. As in the original trial, we found no evidence of an effect of hydroxychloroquine on either transmission or development of symptomatic disease; therefore, we included individuals from both groups of the trial in this study. First, all COVID-19 cases with quantitative PCR data were included in an analysis of the association between clinical and demographic features of cases and viral load. Second, we identified factors associated with transmission using all clusters of an index case (ie, a COVID-19 case with at least one close contact) and their corresponding contacts for which quantitative viral load was available for the index case. Finally, we assessed the risk of developing symptomatic disease and the variation in the incubation period among all contacts with a positive PCR result at baseline, irrespective of available data of their index case.

\section{Study procedures and data collection}

A dedicated outbreak field team visited cases and contacts at home or nursing home on days 1 (enrolment) and 14 . At the first clinical assessment on day 1, the team did a baseline assessment, including a questionnaire for symptoms of COVID-19, and collected relevant epidemiological information by use of a structured interview: time of first exposure to the index case, place of contact (hospital, home, or nursing care facility), routine use of a mask by the contact when in close proximity to the index case and sleep location concerning the index case (eg, same room or same house). Symptom surveillance consisted of active monitoring by phone on days 3 and 7 , a home visit on day 14 , and passive monitoring whenever the participants developed symptoms. Participants who developed symptoms were visited the same day they notified the symptom onset (unscheduled visits) by the field team, who recorded the date of symptom onset, type of symptoms from a prespecified checklist, and symptom severity, graded on a scale of 1 to 4 .

All participants underwent serial SARS-CoV-2 PCR test and viral load titration from nasopharyngeal swabs on day 1 and day 14 and on any unscheduled visit when the participant notified the onset of COVID-19 symptoms (appendix $2 \mathrm{p} \mathrm{1)}$. The detection of SARS-CoV-2 virus was done by PCR from nasopharyngeal swabs at SYNLAB Diagnostics (Barcelona, Spain) by use of the TaqMan 2019-nCoV assay kit according to the manufacturer's protocol (Thermo Fischer Scientific, Waltham, MA, USA). Viral load was quantified from nasopharyngeal swabs at IrsiCaixa laboratory (Badalona, Spain) by PCR amplification, on the basis of the 2019 novel coronavirus realtime RT-PCR diagnostic panel guidelines and protocol developed by the US Centers for Disease Control and Prevention..$^{23}$ For absolute quantification, a standard curve was built using $1 / 5$ serial dilutions of a SARS-CoV-2 plasmid (2019-nCoV_N_Positive Control, $2 \times 10^{5}$ copies

\begin{tabular}{|lc|}
\hline \multicolumn{2}{|c|}{ Value } \\
\hline Cluster size & $2(1-3)$ \\
\hline Index cases $(n=282)$ & $42(13)$ \\
Age, years & \\
Sex & $80(28 \%)$ \\
Men & $202(72 \%)$ \\
Women & $8(6-9)$ \\
Log viral load & \\
Contacts ( $n=753)$ & $42(15)$ \\
Age, years & \\
Sex & $305(41 \%)$ \\
Men & $385(51 \%)$ \\
Women & $63(8 \%)$ \\
Missing & $93(12 \%)$ \\
\hline Baseline positive PCR of contact case & \\
Form of contact & $254(34 \%)$ \\
Health-care worker & $382(51 \%)$ \\
Household & $21(3 \%)$ \\
Nursing home & $96(13 \%)$ \\
Unknown & \\
\hline Tata are $n$ (\%), mean (SD), or median (IQR). Baseline characteristics of linked transmission clusters \\
\hline
\end{tabular}




\begin{tabular}{|c|c|c|c|c|c|}
\hline & $\begin{array}{l}\log _{10} \text { viral load } \\
\text { per } \mathrm{mL}\end{array}$ & $\begin{array}{l}\text { Unadjusted } \\
\beta \text { coefficient }(95 \% \mathrm{Cl})\end{array}$ & $\mathrm{p}$ value & $\begin{array}{l}\text { Adjusted } \\
\beta \text { coefficient }(95 \% \mathrm{Cl})\end{array}$ & $\mathrm{p}$ value \\
\hline Case age & NA & $0.00(-0.02$ to 0.02$)$ & 0.78 & $0.01(-0.01$ to 0.02$)$ & 0.38 \\
\hline \multicolumn{6}{|l|}{ Case sex } \\
\hline Men & 8.15 (7.54 to 8.77$)$ & Reference & .. & Reference & .. \\
\hline Women & 8.04 (7.47 to 8.60$)$ & $-0.24(-0.72$ to 2.40$)$ & 0.33 & $-0.22(-0.61$ to 0.34$)$ & 0.59 \\
\hline $\begin{array}{l}\text { Days from } \\
\text { symptom onset }\end{array}$ & NA & $-0.17(-0.26$ to 0.08$)$ & 0.0002 & $-0.16(-0.24$ to 0.07$)$ & 0.0004 \\
\hline \multicolumn{6}{|l|}{ Symptoms } \\
\hline \multicolumn{6}{|l|}{ Cough } \\
\hline Absent & 7.82 (7.24 to 8.41$)$ & Reference & .. & Reference & .. \\
\hline Present & 8.37 (7.78 to 8.95$)$ & $0.66(0.22$ to 1.10$)$ & 0.0032 & $0.41(-0.02$ to 0.84$)$ & 0.055 \\
\hline \multicolumn{6}{|l|}{ Dyspnoea } \\
\hline Absent & 7.97 (7.50 to 8.43$)$ & Reference & .. & Reference & .. \\
\hline Present & 8.22 (7.45 to 8.99$)$ & $0.27(-0.40$ to 0.94$)$ & 0.42 & $0.28(-0.35$ to 0.92$)$ & 0.38 \\
\hline \multicolumn{6}{|l|}{ Fever } \\
\hline Absent & 7.77 (7.16 to $8 \cdot 38)$ & Reference & .. & Reference & .. \\
\hline Present & 8.42 (7.86 to 8.98$)$ & $0.80(0.36$ to 1.24$)$ & 0.0004 & 0.43 (0.00 to 0.87$)$ & 0.054 \\
\hline \multicolumn{6}{|l|}{ Anosmia } \\
\hline Absent & $8.32(7.76$ to 8.88$)$ & Reference & .. & Reference & .. \\
\hline Present & 7.87 (7.25 to 8.49$)$ & $-0.57(-1.00$ to -0.09$)$ & 0.019 & $-0.54(-1.00$ to 0.09$)$ & 0.019 \\
\hline \multicolumn{6}{|l|}{ Rhinitis } \\
\hline Absent & 7.60 (7.23 to 7.98$)$ & Reference & .. & Reference & .. \\
\hline Present & 8.59 (7.65 to 9.52$)$ & $0.88(-0.05$ to 1.82$)$ & 0.064 & $0.77(-0.11$ to 1.66$)$ & 0.087 \\
\hline \multicolumn{6}{|l|}{ NA=not applicable. } \\
\hline
\end{tabular}

per $\mu \mathrm{L}$, Integrated DNA Technologies, Coralville, IA, USA) and run in parallel to all PCR determinations.

\section{Outcomes and definitions}

Transmission was characterised by examining the number of individuals infected and uninfected among close contacts of an index case. We defined transmission events as PCR positivity at any timepoint (ie, days 1,14 , or at any other unscheduled PCR testing when participants referred symptoms) of a contact in the same household or workplace within 14 days after enrolment. We defined secondary attack rate of viral transmission as the ratio of individuals with a positive PCR test among close contacts, according to WHO guidelines.

Development to symptomatic disease was defined as presence of at least one of the following symptoms: fever, cough, difficulty breathing, myalgia, headache, sore throat, new olfactory and taste disorder, or diarrhoea) and a positive SARS-CoV-2 RT-PCR test. The incubation period was defined as time from first exposure to symptom onset, with later confirmation of infection by PCR..$^{24}$ The earliest possible exposure with the symptomatic index case was determined for each contact individually.

\section{Statistical analysis}

We used log-transformed viral loads that were approximately normally distributed and that also aligned with common reporting norms. The relationship between characteristics of cases and viral load was assessed by use of linear regression considering age (in years), sex, number of days from reported symptom onset, and presence or absence of five key clinical features: fever cough, dyspnoea, rhinitis, and anosmia. To identify risk factors for transmission, we used logistic regression modelling for the risk of transmission using a randomeffect model to allow for within-cluster variation in the risk of transmission. Factors with potential influence on the risk of transmission included characteristics of the potential transmitter (ie, age, sex, viral load, and the presence or absence of respiratory symptoms) and contacts (ie, age, sex, and the type of contact they had with the index case). Finally, the risk of developing symptomatic COVID-19 was assessed by fitting a Cox-regression model considering the age (in years) and sex of the individual, the presence or absence of cardiovascular disease and chronic respiratory disease, and the initial viral load relative to the time to development of symptomatic disease. Data at 14 days after the first study visit were censored, in line with the follow-up done in the original trial. All analyses were done in $\mathrm{R}$, version 4.0.

\section{Role of the funding source}

The funder of the study had no role in the study design, data collection, data analysis, data interpretation, or writing of the report. All authors had full access to all the data in the study and had final responsibility for the decision to submit for publication.

\section{Results}

Between March 17 and April 28, 2020, we identified 314 patients with COVID-19 in whom the viral load was tested. Of these, 220 (70\%) were women, 94 (30\%) were men, and the median age was 41 years (IQR 31-52). Of the 314 patients, $282(90 \%)$ had at least one close contact, resulting in 282 corresponding clusters with a total of 753 contacts. Clusters had a median of two contacts (IQR 1-3) and a maximum of 19 contacts. Most index cases of the clusters were women (202 [72\%]), with an average age of 42 years (SD 13; table 1).

The first study visit was done a median of 4 days (IQR 3-5) after symptom onset. At the first study visit, the median viral load among patients with COVID-19 was $1 \times 110^{8}$ copies per $\mathrm{mL}$ (IQR $1 \times 110^{6}$ to $1 \times 10^{9}$ ). In multivariable linear regression, the viral load among cases was positively associated with the presence of fever and negatively associated with the presence of anosmia (table 2), but no association was observed with age or sex of the COVID-19 case nor with the presence of reported dyspnoea or cough. As anticipated, viral load was negatively associated with the number of days from symptom onset.

For our risk factor analysis on SARS-CoV-2 transmission, we used linked case and contact data of 282 clusters with 753 contacts. At the cluster level, 

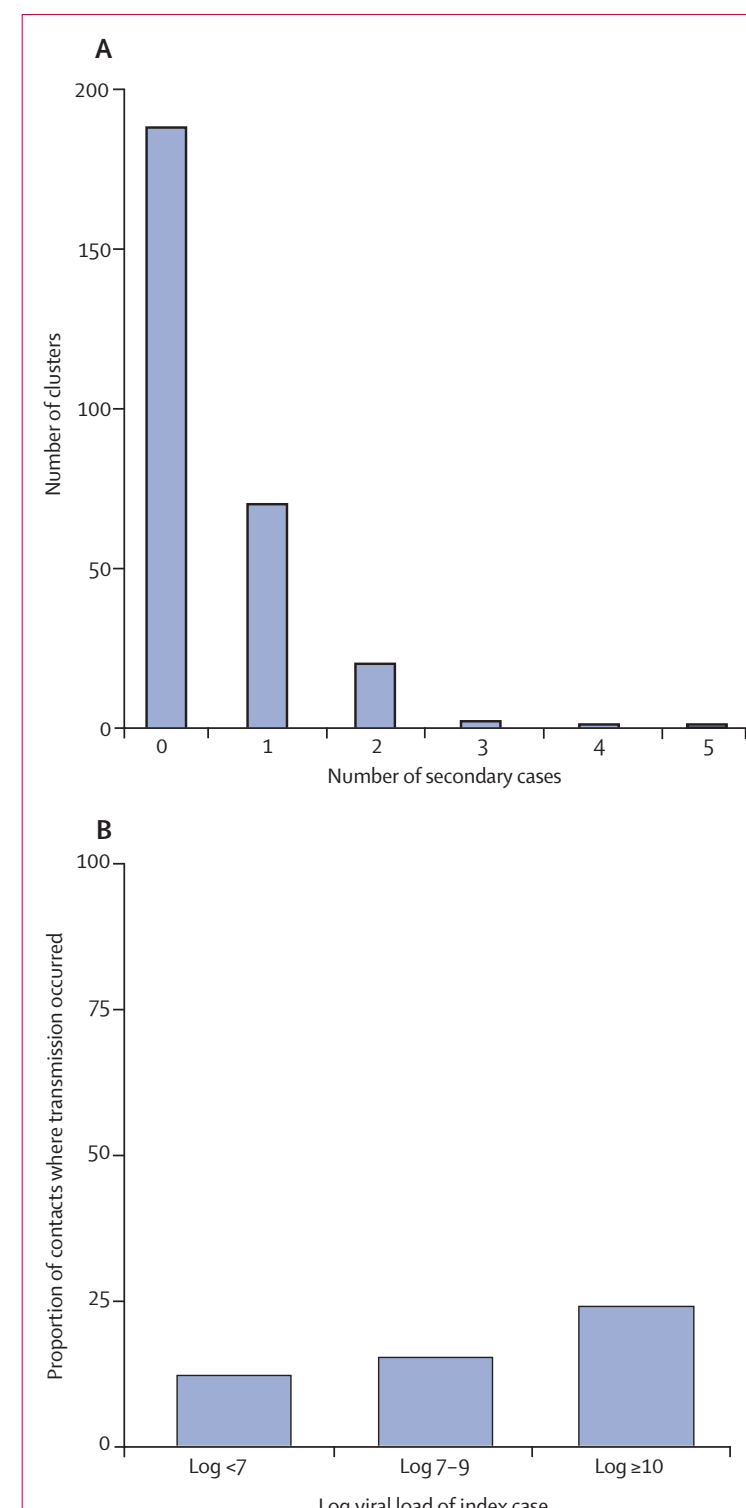

Figure 1: Transmission in a cluster

(A) Number of secondary cases per cluster. (B) Relationship between viral load of the index case and the proportion of contacts developing COVID-19: 36 of 284 contacts in group $<1 \times 110^{7}$ copies per $\mathrm{mL}, 72$ of 398 in group $1 \times 110^{7}$ to $<1 \times 110^{10}$, and 17 of 71 in group $\geq 1 \times 10^{10}$.

$90(32 \%)$ of 282 clusters had at least one transmission event, with a highly skewed distribution of the number of transmission events per cluster (figure 1A). The first visit for contacts took place a median of 5 days (IQR 4-7) after their first possible exposure to the index case. 125 (17\%) of 753 contacts had a PCR positive result over the study period. The proportion of contacts who tested positive for SARS-CoV-2 within a cluster (secondary attack rate) progressively increased with the viral load of the index case: from $12 \%$ when the index case had a viral load lower than $1 \times 10^{6}$ copies per $\mathrm{mL}$ to $24 \%$ when the index case had

\begin{tabular}{|c|c|c|c|c|}
\hline & $\begin{array}{l}\text { Unadjusted odds ratio } \\
(95 \% \mathrm{Cl})\end{array}$ & $p$ value & $\begin{array}{l}\text { Adjusted odds ratio } \\
(95 \% \mathrm{Cl})\end{array}$ & $p$ value \\
\hline \multicolumn{5}{|l|}{ Index case } \\
\hline Age, per year & $1.02(0.99-1.05)$ & 0.069 & $1.01(0.99-1.03)$ & 0.46 \\
\hline \multicolumn{5}{|l|}{ Sex } \\
\hline Men & 1 (ref) & NA & 1 (ref) & NA \\
\hline Women & $0.74(0.40-1.36)$ & 0.33 & $0.71(0.37-1 \cdot 39)$ & 0.32 \\
\hline Viral load, per $\log _{10}$ change & $1.27(1.09-1.48)$ & 0.0020 & $1.29(1.10-1.50)$ & 0.0012 \\
\hline Cough & $1.00(0.55-1.82)$ & 0.99 & $1.13(0.64-2.00)$ & 0.66 \\
\hline Dyspnoea & $0.80(0.31-2.07)$ & 0.64 & $0.75(0.30-1.89)$ & 0.55 \\
\hline Rhinitis & $1.46(0.46-4.63)$ & 0.52 & $1 \cdot 31(0 \cdot 42-4 \cdot 11)$ & 0.64 \\
\hline \multicolumn{5}{|l|}{ Contact } \\
\hline Age, peryear & $1.03(1.01-1.05)$ & 0.0085 & $1.02(1.01-1.04)$ & 0.0008 \\
\hline \multicolumn{5}{|l|}{ Sex } \\
\hline Men & 1 (ref) & NA & 1 (ref) & NA \\
\hline Women & $0.93(0.58-1.49)$ & 0.77 & $1.33(0.79-2 \cdot 23)$ & 0.28 \\
\hline \multicolumn{5}{|l|}{ Mask use by the contact } \\
\hline Never & 1 (ref) & NA & 1 (ref) & NA \\
\hline Always & $0.93(0.47-1.83)$ & 0.84 & $1.55(0.76-3.16)$ & 0.23 \\
\hline Unknown & $1.18(0.59-2 \cdot 36)$ & $0 \cdot 47$ & $1.49(0.74-3.01)$ & 0.26 \\
\hline \multicolumn{5}{|l|}{ Contact type } \\
\hline Health-care work & 1 (ref) & NA & 1 (ref) & NA \\
\hline Household & $3.07(1.68-5.62)$ & 0.0003 & $3.00(1.59-5.65)$ & 0.0006 \\
\hline Nursing home & $1 \cdot 75(0 \cdot 19-16 \cdot 01)$ & 0.62 & $1.90(0.30-1.91)$ & 0.49 \\
\hline Other & $0.32(0.03-3.05)$ & 0.32 & $1 \cdot 19(0 \cdot 10-4 \cdot 31)$ & 0.89 \\
\hline
\end{tabular}

a viral load of $1 \times 10^{10}$ copies per $\mathrm{mL}$ or higher (figure $1 \mathrm{~B}$ ). According to the multivariate analysis, the viral load of the index case was strongly associated with the risk of onward transmission (adjusted odds ratio per $\log _{10}$ increase in viral load $1 \cdot 3,95 \%$ CI $1 \cdot 1-1 \cdot 5$; table 3 ). $114(90 \%)$ of 125 transmission events occurred in clusters where the index case had a viral load of $5 \cdot 1 \log _{10}$ copies per $\mathrm{mL}$ or higher, and $61(50 \%)$ occurred in clusters where the index case had a viral load of $8 \cdot 8 \log _{10}$ copies per $\mathrm{mL}$ or higher. Other factors associated with an increased risk of transmission were household contact (adjusted OR 3.0, $95 \%$ CI $1 \cdot 59-5 \cdot 65)$ and age of the contact $(1 \cdot 02,1 \cdot 01-1 \cdot 04)$. We observed no association of risk of transmission with reported mask usage by contacts, with the age or sex of the index case, or with the presence of respiratory symptoms in the index case at the initial study visit (table 3).

We did not find any evidence of an association between the viral load of the index cases and the first viral load of incident positive results among contacts $(p=0 \cdot 10$, appendix $2 \mathrm{p} 2)$, and this remained true when adjusting for both the day of illness on which the baseline viral load of the index case was measured and the number of days until the contact was enrolled $(\mathrm{p}=0 \cdot 18)$. Additionally, after excluding contacts who were PCR positive at the first study visit, we found no association between the viral load of the index case and 


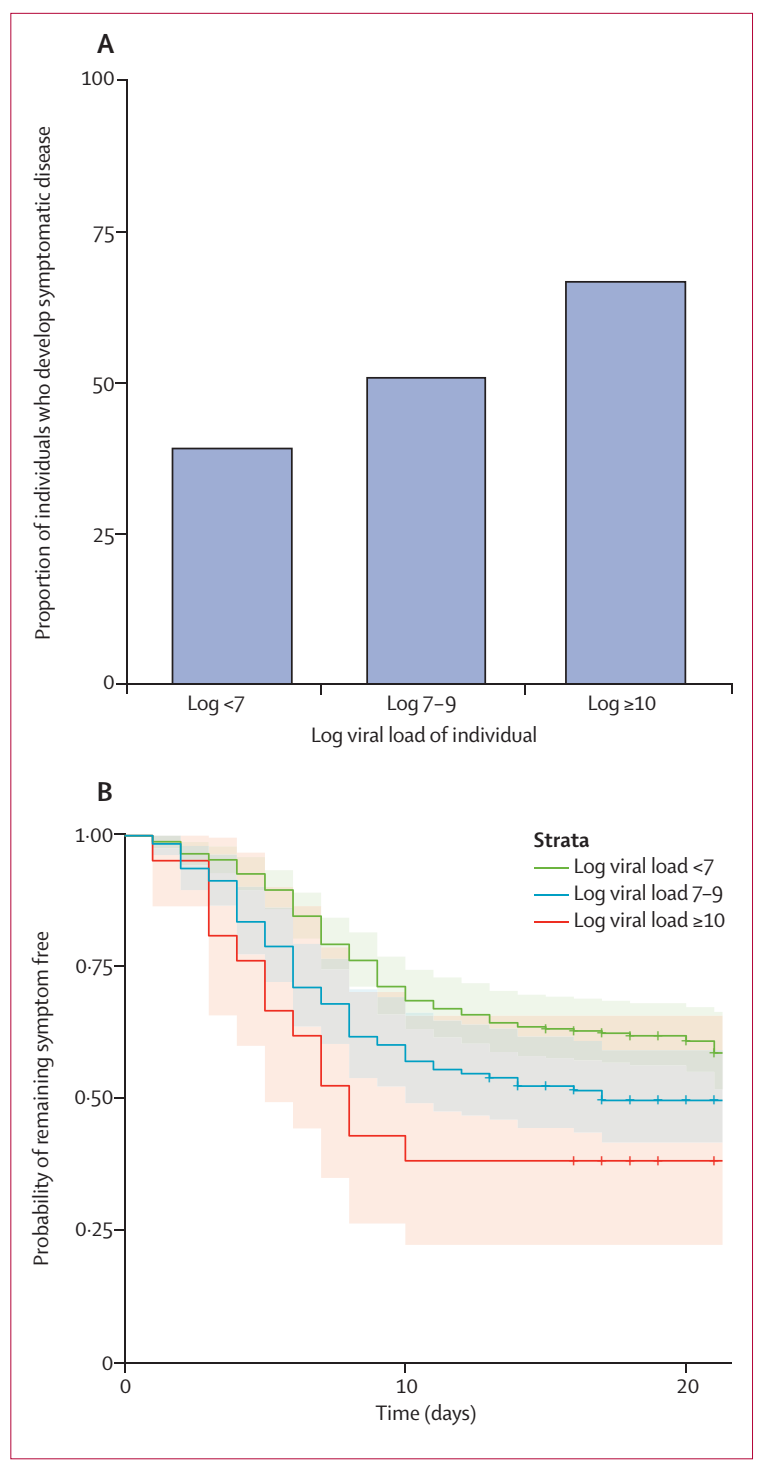

Figure 2: Risk of developing symptomatic COVID-19 according to characteristics of the contact at enrolment

(A) Probability of symptomatic disease by viral load. (B) Time to symptomatic disease by viral load.

the time to onset of incident SARS-CoV-2 infection (hazard ratio [HR] 1.01, 95\% CI 0.83-1.23).

Overall, 449 contacts had a positive PCR result at first visit, whether viral load data of their index case was available $(n=125)$ or not $(n=324) .28(6 \%)$ of 449 contacts had symptoms at the first visit. Of 421 contacts who were asymptomatic at the first visit, 181 (43\%) developed symptomatic COVID-19 within the follow-up period. The multivariable cox-regression analysis, after adjusting for age and sex, showed that increasing viral load levels of the contact at day 1 were associated with an increased risk of developing symptomatic disease. Among contacts not already symptomatic at baseline, the risk of symptomatic disease was approximately $38 \%$ among individuals with an initial viral load lower than $1 \times 10^{7}$ copies per $\mathrm{mL}$ compared with a risk greater than $66 \%$ among those with an initial viral load of $1 \times 110^{10}$ copies per $\mathrm{mL}$ or higher (HR per $\log _{10}$ increase in viral load 1.12, 95\% CI 1.05-1.20; $\mathrm{p}=0.0006$; figure 2A). In the multivariable analysis, no association was found between sex or age of individuals, the presence of diabetes, or presence of cardiovascular or respiratory disease and the risk of or time to developing symptomatic COVID-19.

The median time from exposure to symptom onset was 7 days (IQR 5-9). The time to onset of symptomatic disease decreased from a median of 7 days (5-10) for individuals with an initial viral load lower than $1 \times 10^{7}$ copies per $\mathrm{mL}$ to 6 days (4-8) for those with an initial viral load between $1 \times 10^{7}$ and $1 \times 10^{9}$ copies per $\mathrm{mL}$, and 5 days (3-8) for those with an initial viral load of $1 \times 10^{10}$ copies per $\mathrm{mL}$ or higher (figure $2 \mathrm{~B}$ ). Overall, 110 (61\%) of 181 participants who developed symptoms did so before day $8,45(25 \%)$ between days $8-10$, and $22(12 \%)$ between days 11-14.

\section{Discussion}

In our study, we found that increasing viral load values in nasopharyngeal swabs of patients with COVID-19 were associated with the greater risk of transmission, measured by SARS-CoV-2 PCR positivity among contacts, and with a higher risk of transmission in a household environment compared with that in other indoor situations. Additionally, we found that higher viral loads in swabs of asymptomatic contacts were associated with higher risk of developing symptomatic COVID-19, and that these contacts had shorter incubation periods than those with a lower viral load. Relationships between viral load and infectivity have been described for other respiratory viruses, and our study shows that the same is true for SARS-CoV-2.

To our knowledge, this is the largest study that evaluated the relationship of viral load in patients with COVID-19 and risk of transmission. In our cohort, a high proportion (192 [68\%] of 282) of index cases did not cause secondary infections. However, we identified 90 (32\%) clusters with transmission events, and the multivariate analysis revealed that clusters centred on index cases with high viral load were significantly more likely to result in transmission. In line with previous analyses of case-contact clusters, ${ }^{9,12,14}$ we also found that household exposure to an index case was associated with a higher risk of transmission than other types of contact, presumably reflecting duration and proximity of exposure. Increasing age of the contact was also identified in our multivariate analysis as a significant-albeit modest-determinant of transmission risk. This factor has shown uneven influence across results reported elsewhere but seems to play a secondary role among adults. ${ }^{13,14}$ Finally, unlike previous analyses that reported a relationship between coughing and transmission, ${ }^{13}$ we did not find any association. This finding suggests that 
the absence of cough does not preclude significant onward transmission, particularly if the viral load is high. Taken together, our results indicate that the viral load, rather than symptoms, might be the predominant driver of transmission.

Importantly, we report that high viral load shortly after exposure in asymptomatic contacts was strongly associated with the risk of developing symptomatic COVID-19 disease. We found an approximately $40 \%$ risk of developing symptomatic disease among individuals with an initial viral load lower than $1 \times 10^{7}$ copies per $\mathrm{mL}$ compared with a risk higher than $66 \%$ among individuals with a viral load of $1 \times 110^{10}$ copies per $\mathrm{mL}$ or higher. These data might provide rationale for risk stratification for developing illness. Moreover, the initial viral load significantly shifted the incubation time, which ranged from 5 days in participants with a high viral load to 7 days in participants with a low viral load. To our knowledge, our study was the first analysis of prospective data that investigated the association between initial viral load and incubation time.

The study has several limitations. First, asymptomatic people were not enrolled as index cases, affecting our ability to fully characterise all types of transmission chain. Second, we did not find any evidence of decreased risk of transmission in individuals who reported mask use. Although this finding collides with the evidence reported elsewhere ${ }^{8}$ we did not have fine-grained data on type of mask (surgical vs FFP2) or use of other measures of personal protective equipment (PPE) or other infection control practices, thus limiting our ability to make clear inferences about the effect of PPE on transmission risk. Mask use is probably correlated with type of exposure, which might further confound associations, but we did not note any association between mask use and risk either in our unadjusted analysis (table 3) or in a multivariable model excluding type of exposure (data not shown). Third, we used time to symptom onset (with later confirmation of infection) rather than time to positive PCR test based on serial testing. Nonetheless, accurate calculation of the incubation period was feasible because of the prospective nature of the study, accurate identification of exposure by face-to-face interview, and intensive active and passive monitoring of exposed contacts. We followed up participants over 14-day periods, thus incubation periods longer than 14 days might not have been detected. Within each cluster, we cannot be completely certain about the directionality of transmission, but our inclusion criteria including the absence of COVID-19-like symptoms in the 2 weeks preceding enrolment is consistent with transmission from a case to a contact. We also cannot exclude that some individuals might have been infected by individuals outside of study clusters but, as per national guidelines, all contacts were quarantined after exposure to index cases, reducing the chance of transmission from elsewhere. Samples were available from index cases a median of 4 days after symptom onset, and the initial sample in contacts was taken on average 5 days after exposure, which might limit our ability to detect associations with peak viral load. Despite this, we still showed clear dose effects in relation to both risk of transmission and time to symptom onset. Finally, our study population is reflective of the trial from which the study sample was drawn and is, therefore, biased towards female participants and participants with few comorbidities and predominantly mild to moderate infection; additional data are needed on the risk of transmission in other populations.

In summary, our results provide evidence regarding the determinants of SARS-CoV-2 transmission, particularly on the role of the viral load. The higher risk of transmission among individuals with higher viral loads adds to existing evidence and encourages the assessment of the viral load in patients with a large number of close contacts. When a patient with high viral load is identified, the implementation of reinforced contact tracing measures and quarantines might be crucial to reduce onward transmission. Similarly, our results regarding the risk and expected time to developing symptomatic COVID-19 encourage risk stratification of newly diagnosed SARS-CoV-2 infections on the basis of initial viral load.

\section{Contributors}

$\mathrm{MM}, \mathrm{DO}$, and OM accessed and verified the data. MM, DO, CHR, and OM conceived of the study. MM did the analysis. PM-M, AA, MC-M, MU, MV-M, CG-B, NP, JA, BC, and OM led the randomised controlled trial from which study data are derived. MM and OM wrote the first draft of the manuscript. All authors gave critical input into interpretation and revised the manuscript.

\section{Declaration of interests}

CT reports personal fees from Boehringer Ingelheim and Amgen, outside the submitted work. All other authors declare no competing interests.

\section{Data sharing}

A complete de-identified patient dataset, accompanied by the original trial protocol, will be made available to researchers on request. Individuals wishing to access the data should send a request to mcorbacho@flsida.org. The dataset will be available between Dec 15, 2020, and Dec 15, 2021.

\section{Acknowledgments}

We would like to thank Gerard Carot-Sans for providing medical writing support during the preparation of the manuscript. Our study had support from the crowdfunding campaign YoMeCorono and Generalitat de Catalunya. Support for laboratory equipment was provided by the Foundation Dormeur. ISGlobal receives support from the Spanish Ministry of Science and Innovation through the "Centro de Excelencia Severo Ochoa 2019-2023” Programme (CEX2018-000806-S), and support from the Generalitat de Catalunya through the CERCA Programme. Bàrbara Baro is a Beatriu de Pinós postdoctoral fellow granted by the Government of Catalonia's Secretariat for Universities and Research, and by Marie Sklodowska-Curie Actions COFUND Programme (BP3, 801370).

References

1 La Rosa G, Bonadonna L, Lucentini L, Kenmoe S, Suffredini E. Coronavirus in water environments: occurrence, persistence and concentration methods-a scoping review. Water Res 2020; 179: 115899.

2 Umakanthan S, Sahu P, Ranade AV, et al. Origin, transmission, diagnosis and management of coronavirus disease 2019 (COVID-19). Postgrad Med J 2020; 96: 753-58.

3 Leclerc Q J, Fuller NM, Knight LE, Funk S, Knight GM. What settings have been linked to SARS-CoV-2 transmission clusters? Wellcome Open Res 2020; 5: 83. 
4 Qian H, Miao T. LIU L, Zheng X, Luo D, Li Y. Indoor transmission of SARS-CoV-2. Indoor Air 2020; published online Oct 31. https://doi.org/10.1111/ina.12766

5 Hamner L, Dubbel P, Capron I, et al. High SARS-CoV-2 attack rate following exposure at a choir practice-Skagit County, Washington, March 2020. MMWR Morb Mortal Wkly Rep 2020; 69: 606-10.

6 Park SY, Kim YM, Yi S, et al. Coronavirus disease outbreak in call center, South Korea. Emerg Infect Dis 2020; 26: 1666-70.

7 Muñoz MA, López-Grau M. Lessons learned from the approach to the COVID-19 pandemic in urban primary health care centres in Barcelona, Spain. Eur J Gen Pract 2020; 26: 106-07.

8 Chu DK, Akl EA, Duda S, et al. Physical distancing, face masks, and eye protection to prevent person-to-person transmission of SARS-CoV-2 and COVID-19: a systematic review and meta-analysis. Lancet 2020; 395: 1973-87.

9 Bi Q, Wu Y, Mei S, et al. Epidemiology and transmission of COVID-19 in 391 cases and 1286 of their close contacts in Shenzhen, China: a retrospective cohort study. Lancet Infect Dis 2020; 20: 911-19.

10 Wölfel R, Corman VM, Guggemos W, et al. Virological assessment of hospitalized patients with COVID-2019. Nature 2020; 581: 465-69.

11 La Scola B, Le Bideau M, Andreani J, et al. Viral RNA load as determined by cell culture as a management tool for discharge of SARS-CoV-2 patients from infectious disease wards. Eur J Clin Microbiol Infect Dis 2020; 39: 1059-61.

12 Böhmer MM, Buchholz U, Corman VM, et al. Investigation of a COVID-19 outbreak in Germany resulting from a single travelassociated primary case: a case series. Lancet Infect Dis 2020; 20: 920-28.

13 Wu J, Huang $\mathrm{Y}$, Tu C, et al. Household transmission of SARS-CoV-2, Zhuhai, China, 2020. Clin Infect Dis 2020; published online May 11. https://doi.org/10.1093/cid/ciaa557.

14 Cheng HY, Jian SW, Liu DP, Ng TC, Huang WT, Lin HH. Contact tracing assessment of COVID-19 transmission dynamics in Taiwan and risk at different exposure periods before and after symptom onset. JAMA Intern Med 2020; 180: 1156-63.

15 Huang L, Zhang X, Zhang X, et al. Rapid asymptomatic transmission of COVID-19 during the incubation period demonstrating strong infectivity in a cluster of youngsters aged 16-23 years outside Wuhan and characteristics of young patients with COVID-19: a prospective contact-tracing study. J Infect 2020; 80: e1-13.

16 Liu T, Gong D, Xiao J, et al. Cluster infections play important roles in the rapid evolution of COVID-19 transmission: a systematic review. Int J Infect Dis 2020; 99: 374-80.

17 Buitrago-Garcia D, Egli-Gany D, Counotte MJ, et al. Occurrence and transmission potential of asymptomatic and presymptomatic SARS-CoV-2 infections: a living systematic review and meta-analysis. PLoS Med 2020; 17: e1003346.

18 Backer JA, Klinkenberg D, Wallinga J. Incubation period of 2019 novel coronavirus (2019-nCoV) infections among travellers from Wuhan, China, 20-28 January 2020. Euro Surveill 2020; 25: 20-28.

19 Li Q, Guan X, Wu P, et al. Early transmission dynamics in Wuhan, China, of novel coronavirus-infected pneumonia. N Engl J Med 2020; 382: 1199-207.

20 Leung C. The difference in the incubation period of 2019 novel coronavirus (SARS-CoV-2) infection between travelers to Hubei and nontravelers: the need for a longer quarantine period. Infect Control Hosp Epidemiol 2020; 41: 594-96.

21 Mitjà $\mathrm{O}$, Corbacho-Monné $\mathrm{M}$, Ubals et al. A cluster-randomized trial of hydroxychloroquine for prevention of COVID-19. N Engl J Med 2020; published online Nov 24. https://doi.org/10.1056/ NEJMoa2021801.

22 Catalan Ministry of Health. Catalan epidemiological surveillance system. 2020. http://salutpublica.gencat.cat/ca/ambits/vigilancia salut_publica/ (accessed March 28, 2020; in Catalan).

23 US Centers for Disease Control and Prevention. CDC 2019-novel coronavirus (2019-nCoV) real-time RT-PCR diagnostic panel. Cat. 2019-NCoVEUA-01. 2020. https://www.fda.gov/media/134922/ download (accessed May 21, 2020).

24 WHO. The First Few X (FFX) Cases and contact investigation protocol for 2019-novel coronavirus (2019-nCoV) infection, version 2. https://www.who.int/docs/default-source/coronaviruse/20200129generic-ffx-protocol-2019-ncov.pdf?sfvrsn=595eb313_4 (accessed Sept 21, 2020). 